\title{
ESTUDO COMPARATIVO DA FREQÜÊNCIA DA MORTE SÚBITA INESPERADA POR DOENÇA DE CHAGAS, EM UBERABA, NOS ANOS DE 1980 E 1990
}

\author{
Edison Reis Lopes, Mara Virginia Lellis Marçal, Carlos Siade, Rosemary \\ Kiyoko Asai, Fernando Rodrigues da Cunha Araujo, Lúcio Gaspar \\ Rivas, Renata Ribeiro de Moraes, Vanessa Delfino Dalalio, \\ Adriana Vitor Rezende e Beatriz Hallal Jorge
}

\begin{abstract}
Realizou-se estudo comparativo da freqüência e etiologia das mortes súbitas ocorridas no Município de Uberaba, Minas Gerais, em maiores de 15 anos de idade, nos anos de 1980 e de 1990, com o objetivo fundamental de analisar a freqüência atual do óbito sübito em nossa região e o eventual papel de medidas profilâticas e terapêuticas na morte súbita por doença de Chagas. Dos 1226 óbitos de 1980, 54 (4,4\%) foram súbitos e destes, 13 (24,1\%) etiologia atribuida à doença de Chagas. Em 1990 foram pesquisados 1740 óbitos, dos quais 44 (2,5\%) foram sübitos; destes, somente $3(6,8 \%)$ devidos à tripanosomose cruzi. Os resultados indicam reduçâa significativa na freqüência tanto da morte súbita em geral, como daquela devida à doença de Chagas, no ano de 1990, em relação ao de 1980, discutindo-se suas prováveis razões.
\end{abstract}

Palavras-chaves: Doença de Chagas. Tripanosomose cruzi Morte súbita. Uberaba.

Em 1980, tivemos oportunidade de estudar, entre outros aspectos da morte súbita inesperada (MSI) da doença de Chagas (DC), sua freqüência em ârea endêmica da tripanosomose cruzi. Constatamos ser a cardiopatia chagásica crônica (CCHCr) a causa mais freqüente de óbitos súbitos na cidade de Uberaba, onde realizamos o estudo.

Nos últimos anos, não somente no Brasil Central - onde Uberaba se situa - mas em todo o Brasil e em outros países, foram introduzidas importantes condutas profiláticas contra o $T$. cruzi e novas terapêuticas foram empregadas no tratamento da DC.

Por esta razão, pareceu-nos de interesse averiguar a freqüência da MSI, por DC, dez anos após a análise feita em 1980 e comparar os resultados obtidos nos dois estudos visando a: 1) conhecer a frequuencia atual do óbito súbito do chagásico crônico em nossa região e

Trabalho do Curso de Pós-graduação em Patologia Humana da Faculdade de Medicina do Triângulo Mineiro, Uberaba, MG.

Apoio da Fundação de Amparo à Pesquisa do Estado de Minas Gerais.

Endereço para correspondência: Prof. Edison Reis Lopes. Curso de Pós-graduação em Patologia Humana/FMTM, Av. Getúlio Guaritá s/n, 38025-440 Uberaba, MG, Brasil.

Recebido para publicação em 13/09/94.
2) analisar o papel das citadas medidas profiláticas e terapêuticas na morte súbita (MS) por DC em Uberaba.

\section{MATERIAL E MÉTODOS}

Pesquisaram-se todas as causas de MS, em maiores de 15 anos, constantes dos arquivos do cartório de Registro Civil de Uberaba onde são registrados todos os óbitos ocorridos no Município.

Foram considerados como de MS os óbitos naturais, inesperados, de indivíduos tidos como assintomáticos, nos quais, o tempo decorrido entre o início dos sinais e sintomas e o evento letal, não ultrapassou 24 horas.

Em 1980 foram registados 1618 óbitos, dos quais 1226 ocorreram em maiores de 15 anos de idade. No ano de 1990 foram anotados 1909 falecimentos, 1740 em pessoas com mais de 15 anos.

No exame das certidões de óbito eliminaram-se, preliminarmente, todos os casos relativos a mortes violentas, doenças caquetizantes, degenerativas, neoplasias, processos infecciosos crônicos etc. Os casos de MS e sua etiologia foram confirmados por buscas nos arquivos dos Pronto Socorros e Hospitais e/ou entrevista com médicos e/ou familiares. 
Lopes ER, Marçal MVL, Siade C, Asai RK, Araujo FRC, Rivas LG, Moraes RR, Dalalio VD, Rezende AV, Jorge BH. Estudo comparativo da freqüência da morte sübita inesperada por doença de Cbagas, em Uberaba, nos anos de 1980 e 1990. Revista da Sociedade Brasileira de Medicina Tropical 28:109-112, abr-jun, 1995.

\section{RESULTADOS}

Das 1226 e das 1740 certidões de óbitos analisadas em 1980 e 1990 , respectivamente, $4,4 \%$ e $2,5 \%$ das mortes foram súbitas.
A distribuição das MSI, segundo sua causa, encontra-se na Tabela 1.

Tabela 1 - Causas de mortes sübitas inesperadas em Uberaba em 1980 e 1990, segundo certidōes de óbitos registradas no Cartório Civil.

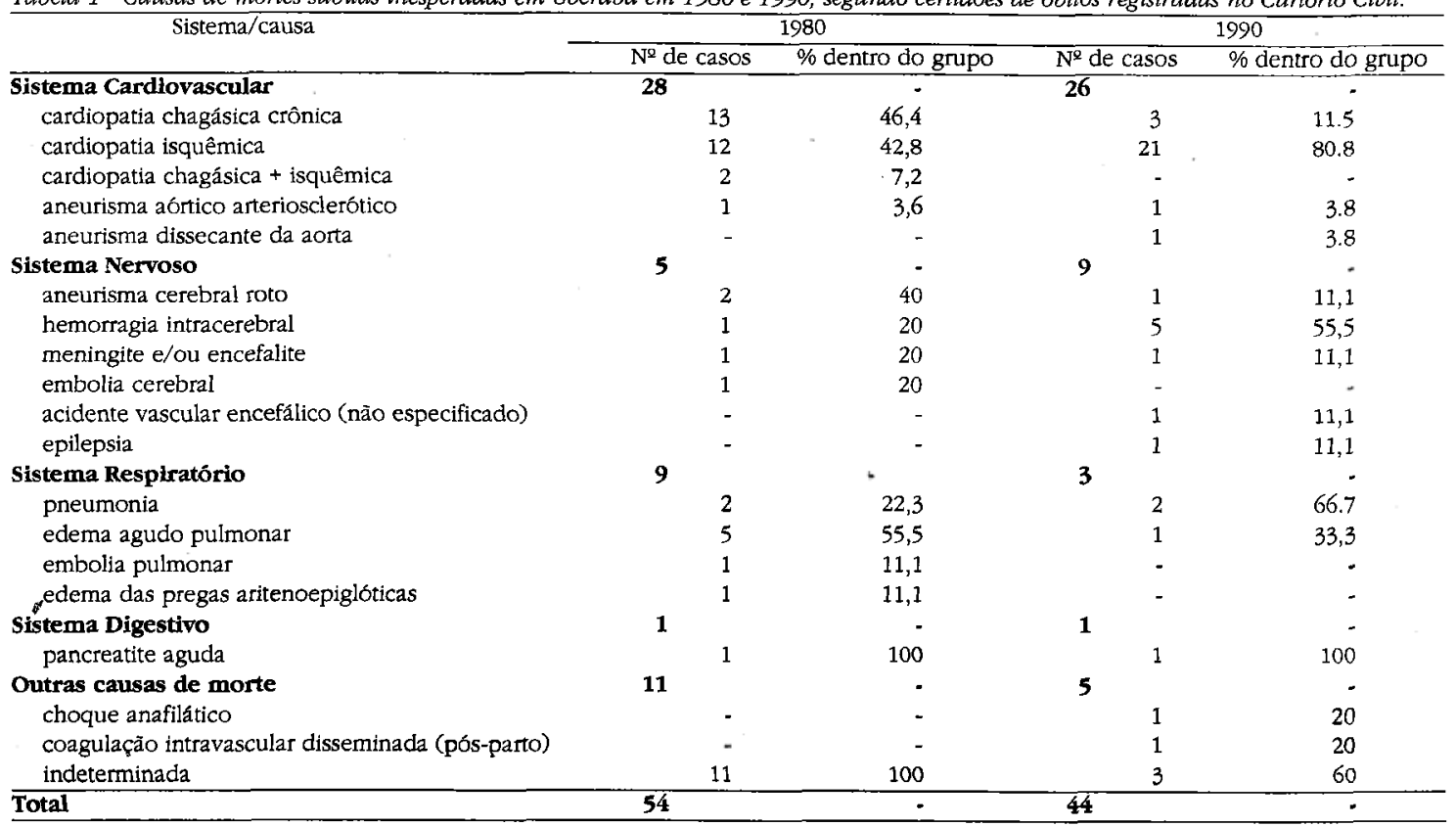

\section{DISCUSSÃO}

A freqüência de $0,17 \%$ de MS por DC constatada, em maiores de 15 anos, falecidos em Uberaba em 1990, é significativamente menor que a de $1,06 \%$ que haviamos detectada, na mesma cidade, em 1980.

Deve-se realçar que nossos dados referemse a casos de MSI, isto é, aquela caracterizada fundamentalmente por sua aparente imprevisibilidade, em pessoas entregues às suas atividades normais ${ }^{1011}$. O conceito de MS esperada e não esperada (inesperada) na DC, somente foi introduzido em 1985, cinco anos após nosso estudo inicial ${ }^{10}$. Tendo em vista o objetivo do atual estudo, os critérios metodológicos empregados para os dados de 1990 foram os mesmos utilizados para os de 1980.

Não temos elementos convincentes para explicar a significativa redução da freqüência da MSI por DC em Uberaba, no ano de 1990, em relação ao de 1980 . Entretanto, podemos aventar algumas hipóteses.
Em primeiro lugar, o fato poderia ser atribuído à eficácia das medidas empregadas na profilaxia da DC. Medidas profiláticas de grande repercussão no combate à $D C$ foram iniciadas na região, em 1950 e reforçadas em 1978. Estas últimas fizeram parte de um plano nacional de combate ao $T$. cruzi e foram bastante eficazes. Contudo, não cremos que a campanha de 1978 se possa atribuir papel na redução da freqüência de MSI por DC observada em Uberaba em 1990. Se considerarmos que a quase totalidade dos chagásicos se infectam nas primeiras décadas de vida $^{4}$ e que a MSI por DC acomete, preferentemente, indivíduos entre 30-40 anos 5 , deduz-se que somente medidas profiláticas implantadas, no mínimo, em 1960/1970, poderiam explicar a redução dos óbitos súbitos em chagásicos constatada em 1990. Quanto à campanha profilática de 1950, como relata Emmanuel Dias" ${ }^{3}$, os trabalhos "não se resumiram a área do Município de Uberaba; estenderam-se à Bambuí e à Cidade Industrial, 
Lopes ER, Marçal MVL, Siade C, Asai RK, Araujo FRC, Rivas LG, Moraes RR, Dalalio VD, Rezende AV, Jorge BH. Estudo comparativo da freqüência da morte sübita inesperada por doença de Chagas, em Uberaba, nos anos de 1980 e 1990. Revista da Sociedade Brasileira de Medicina Tropical 28:109-112, abr-jun, 1995.

bairro proletário de Belo Horizonte". Vê-se, portanto, que esta campanha limitou-se, no Triângulo Mineiro, a Uberabà. Portanto, mesmo que seus resultados tenham sido eficazes - e parece que o foram, como sugerem, entre outros, os dados de Souza' sua contribuição para a redução da MS por DC em Uberaba, em 1990, deve ter sido somente parcial, visto que nossos pacientes nasceram e/ou residiram em municípios outros que não Uberaba, Bambuí e Cidade Industrial.

Dois outros fatores, em nosso entender, podem também ter influído nos resultados. Um deles foi a melhoria das condições habitacionais na zona rural, que apesar de todos os problemas vividos pelo país, sem dúvida, pelo menos em nossa região, foi inquestionável nas últimas décadas. Outro fator é resultado da migração - rural-urbana que carreou grande número de chagásicos da zona rural para as cidades de médio e grande portes entre outras razões pela deficiência de trabalho no campo ${ }^{14}$.

A possibilidade de que medidas terapêuticas possam explicar a redução de MS por DC em 1990 em Uberaba, parece poder ser descartada. A medicação específica é empregada apenas em um número muito reduzido de chagásicos e como analisamos casos de MSI, os pacientes, por não terem conhecimento de sua doença, com certeza não fizeram uso desta terapêutica. Quanto aos antiarrítmicos poder-se-ia argumentar qué a amiodarona que, há alguns anos, vem sendo muito usada no Brasil Central ${ }^{1}$, por ser uma droga capaz de evitar recorrências de taquicardia ventricular e de fibrilação ventricular $^{89}$, poderia, também, prevenir a MS por $\mathrm{DC}^{2}$. Entretanto, por serem os chagásicos de nossa casuística como já se frisou, assintomáticos ou oligossintomáticos, com quase toda probabilidade, não utilizaram antiarrítmicos. Deve-se, ainda, relembrar que não há qualquer pesquisa randômica a respeito de um eventual papel da amiodarona na prèvenção da MS por $\mathrm{DC} \mathrm{e}$, os estudos multicêntricos que analisam o benefício da amiodarona em pacientes com extra-sístoles freqüentes e complexas, não envolvem cardiopatas chagásicos ${ }^{7}$. Finalmente, pode-se ainda associar a esses argumentos, o recente relato de caso de MS por DC descrito nos Estados Unidos da América do Norte, em cardiopata chagásico em uso de procainamida $^{13}$, bem como a descrição de casos de MS em chagásicos em uso de amiodarona ${ }^{7}$.

Outro dado de interesse que nosso estudo mostra é o da diminuição da freqüência do total geral de mortes súbitas, o que se deve provavelmente a redução dos óbitos súbitos na tripanosomose. A percentagem de 2,5 de morte súbita observada em 1990 foi cerca de $56 \%$ menor do que a de $4,4 \%$ detectada em 1980.

Chama também a atenção o aumento da prevalência de MS por cardiopatia isquêmiça (CI) em 1990. Aliás isto é de se esperar em regiôes como a nossa, visto que diminuindo o número de óbitos entre os 35-45 anos, como sucede na tripanosomose cruzi, aumentam as possibilidades de MS por $\mathrm{CI}$, que preferentemente atinge indivíduos em faixas etárias mais elevadas. Mas não é esta provalvemente a única e nem a principal razão que explique a duplicação, por nós constatada, do número de MS por $\mathrm{CI}$, em Uberaba, nos dois anos considerados.

Do ponto de vista epidemiológico, é de interesse comparar a taxa de mortalidade geral, em Uberaba, nos dois períodos considerados. Dados do Instituto Brasileiro de Geografia e Estatística (IBGE) indicam que a população do Município em 1980 - ano no qual ocorreram 1618 óbitos - era de 199.204 habitantes. Já em 1990, quando o Município passou a ter 211.894 habitantes, foram registrados 1909 óbitos. Portanto, as taxas de mortalidade em 1980 e em 1990 foram, respectivamente, 8,12 e 9 por mil, o que é de se esperar, visto que a população do Município teve, em 1990, um aumento de $10,63 \%$ em relação à 1980. Já as MS diminuîram em 18,5\% nos dois períodos considerados.

Ainda dentro do aspecto epidemiológico, haveria interesse em determinar o número de mortes por DC nos dois anos considerados, não só porque com estes dados poderíamos avaliar melhor a importância da MS nestes períodos, mas também compará-los para efeito de medir a tendência do evento sob outro ângulo. Entretanto, a análise que fizessemos sob a fidelidade do diagnóstico de DC firmado nas certidões mostrou a necessidade de que em todos os óbitos, o diagnóstico da tripanosomose tivesse sido confirmado com a mesma metodologia empregada para os casos de MS e isto não foi possível realizar. 
Lopes ER, Marçal MVL, Siade C, Asai RK, Araujo FRC, Rivas LG, Moraes RR, Dalalio VD, Rezende AV, Jorge BH. Estudo comparativo da frequî̀ncia da morte súbita inesperada por doença de Chagas, em Uberaba, nos anos de 1980 e 1990. Revista da Sociedade Brasileira de Medicina Tropical 28:109-112, abr-jun, 1995.

Cremos que as considerações feitas sejam válidas, não só por termos analisado todos os óbitos ocorridos nos dois anos considerados, mas também por sabermos que Rocha observou em Uberlândia (cidade situada a 100 $\mathrm{km}$ de Uberaba) significativa redução da mortalidade por DC nos últimos anos (A. Rocha: comunicação pessoal, 1994).

\section{AGRADECIMENTOS}

A de Registro Civil de Uberaba; ao Prof. Uilho Antonio Gomes, pela orientação na interpretação dos resultados e à Alessandra Scoda pelo auxílio na digitação.

\section{SUMMARY}

A comparison was made between the years 1980 and 1990 for the frequency and causes of sudden death occurring in the urban and rural areas of the city of Uberaba in individuals older than 15 years. It aims mainly to analyse the current frequency of sudden death in that region and to evaluate the impact, it any, of prophylaxis and therapy on sudden death due to Chagas' disease. For the 1226 deaths cases studied from our 1980, 54 (4.4\%) were sudden ones; out of these, 13 (24.1\%) were supposedly due to Chagas' disease. For the 1740 death cases studied form our 1990 series, 44 (2.5\%) were sudden ones; out of these, only 3 (6.8\%) were considered to be due to Chagas' disease. The results indicate a significant decrease in the frequency both for sudden death in general and for sudden death due to Chagas' disease when the year 1990 is compared with 1980. Probable explanations for the findings are discussed.

Key-words: Chagas' disease. Trypanosomiasis cruzi. Sudden deatb. Uberaba.

\section{REFERÊNCIAS BIBLIOGRÁFICAS}

1. Barros MA. Estudo prospectivo dos efeitos da amiodarona na função tiroidiana de pacientes chagásicos em área de deficiência de iodo. Tese doutorado, Escola Paulista de Medicina, São Paulo, 1991.

2. Chiale PA, Halpern MS, Nau GJ,Tambussi AM, Przybylski J, Lázzari JO, Elizari MV, Rosembaum MB. Efficacy of amiodarone during long-term treatment of malignant ventricular arrhythmias in patients with chronic chagasic myocarditis. American Heart Journal 107:656$665,1994$.
3. Dias E. O centro de estudo e profilaxia de moléstia de Chagas em Bambuí, Estado de Minas Gerais. Memórias do Instituto Oswaldo Cruz 54:309-357, 1956.

4. Dias JCP, Cançado JR, Chiari CA. Doença de Chagas. In: Neves J (ed) Diagnóstico e tratamento das doenças infectuosas c parasitárias, $2^{\mathrm{a}}$ edição. Guanabara Koogan, Rio de Janeiro p.694-724, 1983.

5. Lopes ER. Morte súbita em área endêmica da doença de Chagas.Tese Professor Titular, Faculdade de Medicina do Triângulo Mineiro, Uberaba, 1980.

6. Lopes ER, Chapadeiro E. Morte súbita em área endêmica da doença de Chagas. Revista da Sociedade Brasileira de Medicina Tropical 16:79-84, 1983.

7. Mady C, Nacruth R. História natural da cardiopatia chagásica crônica: fatores prognósticos. Revista da Sociedade de Cardiologia do Estado de São Paulo 4:124. 128, 1994.

8. Nademanee K, Hendrickson BS, Cannon DS, Goldneyer BN, Singh BN. Control of refractory life-threatening ventricular tachyarrhythmias by amiodarone. American Heart Journal 101:759-764, 1981.

9. Nademanee K, Hendrickson JA, Kannan R, Singh BN. Antiarrhythmic efficacy and electrophysiologic actions of amiodarone in patients with life-threatening arrhythmias: potent suppression of spontaneously occurring tachyarrhythmias versus inconsistent abolition of induced ventricular tachycardia. American Heart Journal 103:950-957, 1982.

10. Prata A, Lopes ER, Chapadeiro E. Morte súbita na doença de Chagas. In: Cançado JR, Chuster M (ed) Cardiopatia chagásica. Fundação Carlos Chagas, Belo Horizonte, p.114-120, 1985.

11. Prata A, Lopes ER, Chapadeiro E. Características da morte súbita tida como não esperada na doença de Chagas. Revista da Sociedade Brasileira de Medicina Tropical 19:9-12, 1986.

12. Souza HM, Morais CA, Mineo JR. Prevalência da infecção chagásica em doadores de sangue no Triângulo Mineiro. Revista da Sociedade Brasileira de Medicina Tropical 18:11-16, 1985.

13. Scully RE, Mark EJ, McNeely NF, McNeely BU. Weekly clinicopathological exercises. Case 32-1993. New England Journal of Medicine 329:488-496, 1994.

14. Wanderley DMV. Epidemiologia da doença de Chagas. Revista da Sociedade de Cardiologia do Estado de São Paulo 4:77-84, 1994. 